\title{
UNIFORM CONVERGENCE RESULTS FOR CAUCHY PRINCIPAL VALUE INTEGRALS
}

\author{
PHILIP RABINOWITZ
}

\begin{abstract}
A general uniform convergence theorem for numerical integration of Cauchy principal value integrals is proved. Seven special instances of this theorem are given as corollaries.
\end{abstract}

\section{INTRODUCTION}

In this paper we study the uniform convergence with respect to the parameter $\lambda$ of various numerical methods for evaluating the Cauchy principal value $(\mathrm{CPV})$ integral

$$
I(w f ; \lambda):=f_{-1}^{1} w(x) \frac{f(x)}{x-\lambda} d x, \quad-1<\lambda<1,
$$

where $w$ is the Jacobi weight function

$$
w(x):=(1-x)^{\alpha}(1+x)^{\beta}, \quad \alpha, \beta>-1 .
$$

In a previous paper [11], the author showed that if $f$ is Hölder continuous, $f \in H_{\mu}, 0<\mu \leq 1$, where

$$
H_{\mu}:=\left\{g: \omega(g ; t) \leq A t^{\mu}, A>0,0<\mu \leq 1\right\}
$$

and $\omega(g ; t)$ is the modulus of continuity of $g$ on $J:=[-1,1]$,

$$
\omega(g ; t)=\sup _{\substack{\left|x_{1}-x_{2}\right| \leq t \\ x_{1}, x_{2} \in J}}\left|g\left(x_{1}\right)-g\left(x_{2}\right)\right|,
$$

and $\left\{f_{n}\right\}$ is a sequence of piecewise linear approximations to $f$, then

$$
I\left(w r_{n} ; \lambda\right) \rightarrow 0 \text { as } n \rightarrow \infty \text {, uniformly in } \lambda \in(-1,1),
$$

if

$$
\mu+\gamma>0
$$

Received November 16, 1989.

1980 Mathematics Subject Classification (1985 Revision). Primary 65D30.

Key words and phrases. Cauchy principal value integrals, uniform convergence, Hölder continuous functions, Jacobi weight, piecewise polynomials, splines, Lagrange interpolation, Bernstein polynomials, Hermite-Fejér polynomials. 
where $r_{n}(x):=f(x)-f_{n}(x)$ and

$$
\gamma:=\min (\alpha, \beta, 0) \text {. }
$$

Here, we have a sequence of partitions $\Pi_{n}$ given by $\Pi_{n}:-1=x_{0 n}<x_{1 n}<$ $\cdots<x_{p_{n}, n}=1$ with $p_{n+1}>p_{n}, h_{i n}=x_{i+1, n}-x_{i n}$ and $H_{n}=\max _{0 \leq i \leq p_{n}-1} h_{i n}$ and assume that $\lim _{n \rightarrow \infty} H_{n}=0$. The function $f_{n}$ satisfies $f_{n}\left(x_{i n}\right)=f\left(x_{i n}\right)$, $i=0, \ldots, p_{n}$, and is linear on every subinterval $J_{i n}:=\left[x_{i n}, x_{i+1, n}\right]$.

The proof of (3) used the following three properties of $r_{n}$ which were demonstrated in [11]:

(i) $r_{n}( \pm 1)=0$,

(ii) $\left\|r_{n}\right\|=\omega\left(f ; H_{n}\right)$, where $\|g\|:=\max _{x \in J}|g(x)|$,

(iii) $\omega\left(r_{n} ; t\right) \leq C \omega(f ; t)$ for some $C>0$.

In this paper we will extend this result to the case where $f_{n}$ is a generalized piecewise polynomial as defined in [12], a cubic spline interpolating $f$ at equally spaced knots, a modified cubic interpolating spline of deficiency 2 as defined in [9] or a quadratic spline interpolant as described in [10]. We shall also give conditions for (3) to hold when $f_{n}$ is a Lagrange interpolating polynomial, a Hermite-Fejér interpolating polynomial or a Bernstein polynomial. In these cases, the conditions for uniform convergence are weaker than in the previous cases. All these convergence results are corollaries of a general convergence theorem which we give in the next section.

There are some other uniform convergence results in the literature. The strongest are those by Criscuolo and Mastroianni [3, 4] for integration rules based on polynomial approximation. Interestingly enough, their convergence conditions are similar to those given here, as we shall see.

\section{A GENERAL UNIFORM CONVERGENCE THEOREM}

In this section, we shall state and prove a general uniform convergence theorem for CPV integrals. The proof follows along the lines of that in [11].

Theorem 1. Let $f \in H_{\mu}$ on $J$ and assume that $f_{n}$ is an approximation to $f$ such that

(a) $r_{n}( \pm 1)=0$,

(b) $\left\|r_{n}\right\|=O\left(A_{n}^{\nu}\right), 0<\nu \leq \mu$, where $\left\{A_{n}\right\}$ is a sequence of positive numbers such that $\lim _{n \rightarrow \infty} A_{n}=0$,

(c) $\omega\left(r_{n} ; t\right)=O\left(t^{\sigma}\right), 0<\sigma \leq \mu$.

Then (3) holds if

$$
\rho+\gamma>0
$$

where $\rho:=\min (\sigma, \nu)$.

Proof. Using the well-known device of subtracting the singularity (see, e.g., [6, p. 184]), we write

$$
I\left(w r_{n} ; \lambda\right)=\int_{-1}^{1} w(x) \frac{r_{n}(x)-r_{n}(\lambda)}{x-\lambda} d x+r_{n}(\lambda) I(w ; \lambda):=T_{1}+T_{2} .
$$


We now show that $T_{1}=T_{1}(\lambda)$ and $T_{2}=T_{2}(\lambda)$ both converge uniformly to 0 for all $\lambda \in(-1,1)$ if $(5)$ holds.

Consider first $T_{2}:=r_{n}(\lambda) I(w ; \lambda)$. Since $r_{n}(1)=0$, we have $r_{n}(\lambda) \leq$ $\omega\left(r_{n} ; 1-\lambda\right)=O\left((1-\lambda)^{\sigma}\right)$. Furthermore, in a neighborhood of $\lambda=1$,

\section{$[13, \S 4.62]$.}

$$
I(w ; \lambda)= \begin{cases}O\left((1-\lambda)^{\alpha}\right)+C & \text { if } \alpha \text { is not an integer }, \\ O(|\log (1-\lambda)|) & \text { if } \alpha \text { is an integer }\end{cases}
$$

Hence, we can find $s>0$ sufficiently small so that for all $\lambda$ in $[1-s, 1]$

$$
T_{2}=O\left((1-\lambda)^{\sigma+\alpha}|\log (1-\lambda)|\right)<\varepsilon
$$

uniformly in $\lambda$ if (5) holds. Similarly, we can find $\bar{s}>0$ such that for all $\lambda$ in $[-1,-1+\bar{s}]$

$$
T_{2}=O\left((1+\lambda)^{\sigma+\beta}|\log (1+\lambda)|\right)<\varepsilon
$$

uniformly in $\lambda$. Finally, since $I(w ; \lambda)=O(1)$ in $[-1+\bar{s}, 1-s]$ and $\left\|r_{n}\right\|=$ $o(1)$ as $n \rightarrow \infty$, we conclude that $T_{2}=o(1)$ uniformly in $\lambda$ as $n \rightarrow \infty$.

We now turn to $T_{1}$, which we write as

$$
T_{1}=\int_{U} h_{n}(x) d x+\int_{\substack{|x-\lambda| \geq A_{n} \\ x \notin U}} h_{n}(x) d x+\int_{\substack{|x-\lambda| \leq A_{n} \\ x \notin U}} h_{n}(x) d x:=I_{1}+I_{2}+I_{3} \text {, }
$$

where $h_{n}(x):=w(x)\left(r_{n}(x)-r_{n}(\lambda)\right) /(x-\lambda)$ and $U:=[-1,-1+r] \cup[1-\bar{r}, 1]$ for some $r, \bar{r}$ to be determined below.

Consider now the integral

$$
\begin{aligned}
& \left|\int_{-1}^{-1+r} h_{n}(x) d x\right|=O\left(\int_{-1}^{-1+r}(1+x)^{\beta}|x-\lambda|^{\sigma-1} d x\right) \\
& \quad=O\left(\int_{-1}^{-1+r}(1+x)^{\gamma+\sigma-1} d x\right)<\varepsilon \text { for } r \text { sufficiently small. }
\end{aligned}
$$

Similarly, $\left|\int_{1-\bar{r}}^{1} h_{n}(x) d x\right|<\varepsilon$ for $\bar{r}$ sufficiently small, so that $\left|I_{1}\right|<2 \varepsilon$. As for $I_{2}$,

$$
\begin{aligned}
\left|\int_{\substack{|x-\lambda| \geq A_{n} \\
x \notin U}} h_{n}(x) d x\right| & \leq \max _{x \in J-U} w(x) \cdot 2\left\|r_{n}\right\| \int_{\substack{|x-\lambda| \geq A \\
x \notin U}}|x-\lambda|^{-1} d x \\
& =O\left(A_{n}^{\nu}\left|\log A_{n}\right|\right)=o(1) \quad \text { as } n \rightarrow \infty .
\end{aligned}
$$

Finally,

$$
\begin{aligned}
\left|\int_{\substack{|x-\lambda| \leq A_{n} \\
x \notin U}} h_{n}(x) d x\right|=O\left(\int_{\substack{|x-\lambda| \leq A_{n} \\
x \notin U}} \frac{\omega\left(r_{n},|x-\lambda|\right)}{|x-\lambda|} d x\right) \\
=O\left(\int_{\substack{|x-\lambda| \leq A_{n} \\
x \notin U}}|x-\lambda|^{\sigma-1} d x\right) \\
=o(1) \quad \text { as } n \rightarrow \infty \text { uniformly in } \lambda, \text { since } A_{n}=o(1) .
\end{aligned}
$$


Hence, $I\left(w r_{n} ; \lambda\right)$ can be made arbitrarily small as $n \rightarrow \infty$, uniformly in $\lambda \in$ $(-1,1)$.

\section{Particular examples of Theorem 1}

In this section, we derive uniform convergence results for a variety of approximations $f_{n}$ to $f$ which we state as a series of corollaries.

Corollary 1. Let $f \in H_{\mu}$ and let $\left\{f_{n}\right\}$ be a sequence of piecewise polynomials defined as follows: For every partition $\Pi_{n}$, we define a partition $\Pi_{\text {in }}$ of each subinterval $J_{i n}, i=0, \ldots, p_{n}-1$, by

$$
\Pi_{i n}: x_{i n}=x_{i 0}^{(n)}<x_{i 1}^{(n)}<\cdots<x_{i, m_{n i}}^{(n)}=x_{i+1, n}
$$

subject to the conditions $m_{n i} \leq M$ for all $i$ and $n$ and $x_{i, j+1}^{(n)}-x_{i j}^{(n)} \geq d h_{i n}$ for some $d>0$ and all $i, j$, and $n . f_{n}(x)$ is defined on $J_{i n}$ as the Lagrange interpolating polynomial of degree $m_{n i}$ agreeing with $f(x)$ at the points $x_{i j}^{(n)}$, $j=0,1, \ldots, m_{n i}$. Then (3) holds if (4) holds and if $H_{n} \rightarrow 0$ as $n \rightarrow \infty$.

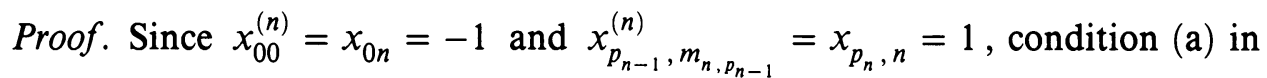
Theorem 1 holds. We show condition (b) with $A_{n}=H_{n}$ and $\nu=\mu$ by writing

$$
f_{n}(x)=\sum_{k=0}^{m_{n i}} l_{i k}^{(n)}(x) f\left(x_{i k}^{(n)}\right), \quad x \in J_{i n},
$$

where

$$
l_{i k}^{(n)}(x)=\prod_{\substack{j=0 \\ j \neq k}}^{m_{n i}} \frac{x-x_{i j}^{(n)}}{x_{i k}^{(n)}-x_{i j}^{(n)}},
$$

which implies that $\left|l_{i k}^{(n)}(x)\right| \leq d^{-M}$ for all $i, k$ and $n$ and all $x \in J$. Hence,

$$
\left|r_{n}(x)\right|=\left|\sum_{k=0}^{m_{n i}}\left(f(x)-f\left(x_{i k}^{(n)}\right)\right) l_{i k}^{(n)}(x)\right| \leq(M+1) d^{-M} H_{n}^{\mu}
$$

as asserted. Finally, we show condition (c) with $\sigma=\mu$ as follows: Using the Newton divided difference form for the interpolating polynomial, we have that, for any $t \in J_{i n}$,

$$
\begin{aligned}
f_{n}(t)= & f\left(x_{i 0}^{(n)}\right)+P_{1}(t) f\left[x_{i 0}^{(n)}, x_{i 1}^{(n)}\right]+P_{2}(t) f\left[x_{i 0}^{(n)}, x_{i 1}^{(n)}, x_{i 2}^{(n)}\right] \\
& +\cdots+P_{m_{n i}}(t) f\left[x_{i 0}^{(n)}, x_{i 1}^{(n)}, \ldots, x_{i, m_{n i}}^{(n)}\right]
\end{aligned}
$$

where

$$
P_{j}(t):=\prod_{k=0}^{j-1}\left(x-x_{i k}^{(n)}\right), \quad j=1, \ldots, m_{n i} .
$$

Since all the zeros of $P_{j}^{\prime}(t)$ lie in $J_{i n}$, we have that

$$
\left|P_{j}^{\prime}(\xi)\right| \leq j h_{i n}^{j-1}, \quad j=1, \ldots, m_{n i} ; \quad \xi \in J_{i n} .
$$


We now show by induction that if $\omega(f ; t) \leq A t^{\mu}$ for some $A>0$, then for any distinct values $y_{j}$ such that

$$
\left\{y_{1}, \ldots, y_{k}\right\} \subset\left\{x_{i 0}^{(n)}, x_{i 1}^{(n)}, \ldots, x_{i, m_{n i}}^{(n)}\right\}, \quad k \geq 2,
$$

we have

$$
\left|f\left[y_{1}, \ldots, y_{k}\right]\right| \leq A 2^{k-2} d^{-k+1} h_{i n}^{\mu-k+1} .
$$

Indeed, for $k=2$

$$
\left|f\left[y_{1}, y_{2}\right]\right|=\left|f\left(y_{1}\right)-f\left(y_{2}\right)\right| /\left|y_{1}-y_{2}\right| \leq A h_{i n}^{\mu} / d h_{i n}=A d^{-1} h_{i n}^{\mu-1},
$$

and for $k>2$

$$
\begin{aligned}
& \left|f\left[y_{1}, y_{2}, \ldots, y_{k}\right]\right|=\left|f\left[y_{1}, \ldots, y_{k-1}\right]-f\left[y_{2}, \ldots, y_{k}\right]\right| /\left|y_{1}-y_{k}\right| \\
& \quad \leq 2\left(A 2^{k-3} d^{-k+2} h_{i n}^{\mu-k+2}\right) / d h_{i n}=A 2^{k-2} d^{-k+1} h_{i n}^{\mu-k+1} .
\end{aligned}
$$

Consider now $u, v \in J_{i n}, u<v$. Then

$$
\begin{aligned}
& f_{n}(v)-f_{n}(u)=(v-u)\left\{P_{1}^{\prime}\left(\xi_{1}\right) f\left[x_{i 0}^{(n)}, x_{i 1}^{(n)}\right]\right.+P_{2}^{\prime}\left(\xi_{2}\right) f\left[x_{i 0}^{(n)}, x_{i 1}^{(n)}, x_{i 2}^{(n)}\right] \\
&\left.+\cdots+P_{m_{n i}}^{\prime}\left(\xi_{m_{n i}}\right) f\left[x_{i 0}^{(n)}, \ldots, x_{i, m_{n i}}^{(n)}\right]\right\}, \\
& u<\xi_{j}<v .
\end{aligned}
$$

Using the bounds (6) and (7), we see that

$$
\begin{aligned}
\left|f_{n}(v)-f_{n}(u)\right| & \leq(v-u) A\left[d^{-1}+2 d^{-2}+\cdots+2^{m_{n i}-1} d^{-m_{n}}\right] h_{i n}^{\mu-1} \\
& \leq B|v-u|^{\mu},
\end{aligned}
$$

where $B:=A\left[d^{-1}+2 d^{-2}+\cdots+2^{M-1} d^{-M}\right]$.

If $u \in J_{i n}, v \in J_{j n}, i<j$, then

$$
f_{n}(v)-f_{n}(u)=f_{n}(v)-f_{n}\left(x_{j n}\right)+f_{n}\left(x_{j n}\right)-f_{n}\left(x_{i+1, n}\right)+f_{n}\left(x_{i+1, n}\right)-f_{n}(u) .
$$

Since $f_{n}\left(x_{k n}\right)=f\left(x_{k n}\right)$ for all $k$, we have that

$$
\begin{aligned}
\left|f_{n}(v)-f_{n}(u)\right| & \leq B\left|v-x_{j n}\right|^{\mu}+A\left|x_{j n}-x_{i+1, n}\right|^{\mu}+B\left|x_{i+1, n}-u\right|^{\mu} \\
& \leq 3 B|v-u|^{\mu} .
\end{aligned}
$$

Finally,

$$
\begin{aligned}
\left|r_{n}(v)-r_{n}(u)\right| & \leq|f(v)-f(u)|+\left|f_{n}(v)-f_{n}(u)\right| \\
& \leq A|v-u|^{\mu}+3 B|v-u|^{\mu} \leq 4 B|v-u|^{\mu},
\end{aligned}
$$

establishing condition (c). This proves the corollary.

Corollary 2. Let $f \in H_{\mu}$ and let $\left\{f_{n}\right\}$ be a sequence of cubic splines with knots $t_{i n}=-1+2 i /(n+1), i=0,1, \ldots, n+1$, which interpolate $f$ at all the knots and also at the points $\frac{1}{2}\left(t_{0 n}+t_{1 n}\right)$ and $\frac{1}{2}\left(t_{n n}+t_{n+1, n}\right)$. Then (3) holds if (4) holds.

Proof. Since $f_{n}$ interpolates $f$ at $t_{0 n}=-1$ and $t_{n+1, n}=1$, condition (a) of Theorem 1 holds. By Lemma 1 in [5], $\left\|r_{n}\right\|=O\left(\omega\left(f ; n^{-1}\right)\right)$, so that condition 
(b) holds with $A_{n}=n^{-1}$ and $\nu=\mu$. By Lemma 4 in [5], $\omega\left(r_{n} ; t\right)=O\left(n^{-\mu+\tau} t^{\tau}\right)$ for any positive $\tau<\mu$. Hence, by condition (c) in Theorem 1, (3) holds if $\tau+\gamma>0$. However, if (4) holds, we can find a positive $\tau<\mu$ such that $\tau+\gamma>0$. Hence, it follows that (3) holds if (4) holds, as asserted.

Corollary 3. Let $f \in H_{\mu}$ and let $\left\{f_{n}\right\}$ be a sequence of modified interpolating cubic splines of deficiency 2 defined on a sequence of partitions $\left\{\Pi_{n}\right\}$ as follows [9]: For any $\Pi_{n}$, let $g_{n}$ be the piecewise linear function defined in $\S 1$. Now, for every $i, i=1, \ldots, p_{n}-1$, choose points $t_{i}^{L} \in J_{i-1, n}, t_{i}^{R} \in J_{i, n}$ such that for some positive $\kappa \leq 1 / 2$

$$
\left|x_{i n}-t_{i}^{L}\right|=\left|x_{i n}-t_{i}^{R}\right|=\kappa \min \left(h_{i-1, n}, h_{i n}\right) .
$$

Let $S_{i}(x)$ be defined on $\left[t_{i}^{L}, t_{i}^{R}\right]$ as the cubic Hermite interpolating polynomial satisfying

$$
\begin{aligned}
S_{i}\left(t_{i}^{L}\right) & =g_{n}\left(t_{i}^{L}\right), & S_{i}^{\prime}\left(t_{i}^{L}\right) & =g_{n}^{\prime}\left(t_{i}^{L}\right), \\
S_{i}\left(t_{i}^{R}\right) & =g_{n}\left(t_{i}^{R}\right), & S_{i}^{\prime}\left(t_{i}^{R}\right) & =g_{n}^{\prime}\left(t_{i}^{R}\right) .
\end{aligned}
$$

Then $f_{n}$ is defined by

$$
f_{n}(x)= \begin{cases}S_{i}(x), & x \in\left[t_{i}^{L}, t_{i}^{R}\right], \quad i=1, \ldots, p_{n}-1, \\ g_{n}(x) & \text { otherwise. }\end{cases}
$$

If $H_{n} \rightarrow 0$ as $n \rightarrow 0$ then (3) holds if (4) holds.

Proof. Since $f_{n}( \pm 1)=g_{n}( \pm 1)=f( \pm 1)$, condition (a) of Theorem 1 holds. By equation (5.1) in [9], $\left\|r_{n}\right\|=O\left(H_{n}^{\mu}\right)$, so that condition (b) holds with $A_{n}=H_{n}$ and $\nu=\mu$. Finally, by equation (5.3) in [9],

$$
\left|r_{n}(x)-r_{n}(y)\right| \leq C H_{n}^{\mu-\tau}|x-y|^{\tau}
$$

for any positive $\tau<\mu$. Hence, our conclusion follows as in the proof of Corollary 2.

Corollary 4. Let $f \in H_{\mu}$ and let $\left\{\Pi_{n}\right\}$ be a sequence of partitions. Let $\lambda_{\text {in }} \in$ $[d, 1-d], i=0, \ldots, p_{n}-1$, for a fixed $d, 0<d \leq 1 / 2$, and define $t_{i n}:=$ $\lambda_{i n} x_{i n}+\left(1-\lambda_{i n}\right) x_{i+1, n}$. Let $f_{n}$ be the quadratic spline defined for $x \in J_{i n}$ by

$$
f_{n}(x):=\left(1-C_{i n}(x)\right) f\left(x_{i n}\right)+C_{i n}(x) f\left(x_{i+1, n}\right)+\left(x-x_{i n}-h_{i n} C_{i n}(x)\right) a,
$$

where $a$ is an arbitrary real number and

$$
C_{i n}(x):= \begin{cases}\left(x-x_{i n}\right)^{2} /\left(1-\lambda_{i n}\right) h_{i n}^{2}, & x_{i n} \leq x \leq t_{i n}, \\ 1-\left(x_{i+1, n}-x\right)^{2} / \lambda_{i n} h_{i n}^{2}, & t_{i n} \leq x \leq x_{i+1, n} .\end{cases}
$$

Then (3) holds if (4) holds and if $H_{n} \rightarrow 0$ as $n \rightarrow \infty$.

Proof. Since $f_{n}$ interpolates $f$ at all points in $\Pi_{n}$, condition (a) of Theorem 1 holds. By Neuman and Schmidt [10, Theorem 4.2],

$$
\left\|f-f_{n}\right\| \leq|a| H_{n} / 4+\omega\left(f ; H_{n}\right)=O\left(H_{n}^{\mu}\right),
$$


so that condition (b) holds with $A_{n}=H_{n}$ and $\nu=\mu$. To show condition (c) with $\sigma=\mu$, we assume that $u<v$ and examine first the case $u, v \in\left[x_{i n}, t_{i n}\right]$ for some $i$. Then,

$$
\begin{aligned}
f_{n}(v)-f_{n}(u)= & \left(C_{i n}(v)-C_{i n}(u)\right)\left(f\left(x_{i+1, n}\right)-f\left(x_{i n}\right)\right) \\
& +a\left[(v-u)-h_{i n}\left(C_{i n}(v)-C_{i n}(u)\right)\right] .
\end{aligned}
$$

But

Hence,

$$
\begin{aligned}
\left|C_{i n}(v)-C_{i n}(u)\right| & =\left|(v-u)\left(v+u-2 x_{i n}\right) /\left(1-\lambda_{i n}\right) h_{i n}^{2}\right| \\
& \leq 2 d^{-1}(v-u) / h_{i n} .
\end{aligned}
$$

$$
\left|f_{n}(v)-f_{n}(u)\right| \leq B_{1}(v-u) h_{i n}^{\mu-1}+B_{2}(v-u)=O\left(|v-u|^{\mu}\right)
$$

and similarly if $u, v \in\left[t_{i n}, x_{i+1, n}\right]$. For $u, v \in J_{\text {in }}, u \leq t_{\text {in }} \leq v$, we write

$$
f_{n}(v)-f_{n}(u)=f_{n}(v)-f_{n}\left(t_{\text {in }}\right)+f_{n}\left(t_{\text {in }}\right)-f_{n}(u)
$$

and get the same result. For the case $u \in J_{i n}, v \in J_{j n}, i<j$, and the rest of the proof, refer to the proof of Corollary 1 .

Corollary 5. Let $f \in H_{\mu}$ and let $\left\{f_{n}\right\}$ be the sequence of Bernstein polynomials

$$
f_{n}(x):=2^{-n} \sum_{k=0}^{n} f(-1+2 k / n)\left(\begin{array}{l}
n \\
k
\end{array}\right)(1+x)^{k}(1-x)^{n-k} \text {. }
$$

Then (3) holds if

$$
\mu / 2+\gamma>0 \text {. }
$$

Proof. Clearly, $f( \pm 1)=0$. Furthermore, by Theorem 1 in [1], condition (c) holds with $\sigma=\mu$. Finally, by Theorem 1.6.1 in [8], $\left\|r_{n}\right\|=O\left(n^{-\mu / 2}\right)$, so that condition (b) holds with $A_{n}=n^{-1}$ and $\nu=\mu / 2$.

Corollary 6. Let $f \in H_{\mu}$, let $\left\{X_{n}\right\}$ be a sequence of point sets defined by

$$
X_{n}:-1=x_{0 n}<x_{1 n}<\cdots<x_{n n}=1
$$

with Lebesgue constants $\Lambda\left(X_{n}\right)$ with respect to Lagrange interpolation, and let $\left\{f_{n}\right\}$ be the sequence of Lagrange interpolation polynomials interpolating $f$ on the sets $X_{n}$. If $\Lambda\left(X_{n}\right)=O(\log n)$, then (3) holds if (8) holds. If $\Lambda\left(X_{n}\right)=O\left(n^{\tau}\right)$ for some $\tau>0$, then (3) holds if $\mu-\tau+2 \gamma>0$.

Proof. Since $x_{0 n}=-1$ and $x_{n n}=1$ for all $n$, we have $r_{n}( \pm 1)=0$. Furthermore, we have that

$$
\left\|r_{n}\right\| \leq\left(1+\Lambda\left(X_{n}\right)\right) E_{n} f,
$$

where $E_{n} f=\left\|f-q_{n}\right\|$ and $q_{n}$ is the polynomial of degree $n$ of best approximation to $f$ in the uniform norm.

We consider first the case $\Lambda\left(X_{n}\right)=O(\log n)$. Since by Jackson's theorem, $E_{n} f=O\left(n^{-\mu}\right)$, it follows that $\left\|r_{n}\right\|=O\left(n^{-\mu_{1}}\right)$ for any positive $\mu_{1}<\mu$. Now, by Kalandiya's theorem (see, e.g., [7, Lemma 1]), we have that

$$
\omega\left(r_{n} ; t\right)=O\left(t^{\mu_{2} / 2}\right)
$$


for any positive $\mu_{2}<\mu_{1}$. Hence, by Theorem 1, (3) holds if $\mu_{2} / 2+\gamma>0$. However, if (8) holds, we can find $\mu_{1}, \mu_{2}$ such that $0<\mu_{2}<\mu_{1}<\mu$ and such that $\mu_{2} / 2+\gamma>0$.

If $\Lambda\left(X_{n}\right)=O\left(n^{\tau}\right)$, then $\left\|r_{n}\right\|=O\left(n^{-\mu+\tau}\right)$, so that by Kalandiya's theorem, $\omega\left(r_{n} ; t\right)=O\left(t^{\sigma}\right)$ with $\sigma<(\mu-\tau) / 2$. The rest of the proof proceeds as before.

Remark. Two examples of sets $X_{n}$ such that $\Lambda\left(X_{n}\right)=O(\log n)$ are as follows:

(1) $x_{i n}$ are the zeros of $\left(1-x^{2}\right) P_{n-1}^{(\bar{\alpha}, \bar{\beta})}(x)$, where $P_{n-1}^{(\bar{\alpha}, \bar{\beta})}$ is the Jacobi polynomial of degree $n-1$ and $-1 / 2 \leq \bar{\alpha}, \bar{\beta} \leq 3 / 2$ [15].

(2) $x_{i n}=\sec (\pi /(2 n+2)) \cos [\pi-(2 i+1) \pi /(2 n+2)], i=0, \ldots, n$, the so-called extended Chebyshev nodes [2].

Corollary 7. Let $f \in H_{\mu}$ and let $f_{n}=H_{n p q}(f), p, q \geq 1$, be the Hermite-Fejér interpolation polynomial with boundary conditions based on the zeros $\left\{x_{i n}, i=\right.$ $1, \ldots n\}$ of the Jacobi polynomial $P_{n}^{\hat{\alpha}, \hat{\beta}}$, which satisfy the following conditions:

$$
\begin{aligned}
& H_{n p q}\left(f ; x_{i n}\right)=f\left(x_{i n}\right), H_{n p q}^{\prime}\left(f ; x_{i n}\right)=0, i=1, \ldots, n, \\
& H_{n p q}(f ; \pm 1)=f( \pm 1), \\
& H_{n p q}^{(r)}(f ; 1)=0, r=1, \ldots, p-1, H_{n p q}^{(s)}(f ;-1)=0, s=1, \ldots, q-1 .
\end{aligned}
$$

If $p-1.5 \leq \hat{\alpha} \leq p-.5, q-1.5 \leq \hat{\beta} \leq q-.5$, then (3) holds when (8) holds. Proof. By Vértesi [14, Section 3.4.3],

$$
\left|r_{n}(x)\right|=O(1) \sum_{i=1}^{n}\left[\omega\left(f, \frac{i \sin \theta}{n}\right)+\omega\left(f, \frac{i^{2}|\cos \theta|}{n^{2}}\right)\right] i^{-2},
$$

where $x=\cos \theta$. This implies that $\left\|r_{n}\right\|=O\left(n^{-\mu}\right)$ when $\mu<1$ and $\left\|r_{n}\right\|=$ $O(\log n / n)$ when $\mu=1$. Since $r_{n}( \pm 1)=0$, we can proceed as in the proof of the first part of Corollary 6 .

\section{OTHER UNIFORM CONVERGENCE RESULTS}

Criscuolo and Mastroianni [3] consider the CPV integral $I(\bar{w} f ; \lambda)$, where

$$
\bar{w}(x):=\psi(x) w(x)
$$

and $\psi(x)>0$ on $J$ and satisfies

$$
\int_{0}^{2} \omega(\psi ; t) t^{-1} d t<\infty
$$

Since $I(\bar{w} f ; \lambda)=\int_{-1}^{1} \bar{w}(x) \frac{f(x)-f(\lambda)}{x-\lambda} d x+I(\bar{w} ; \lambda)$, they consider the approximation to $I(\bar{w} f ; \lambda)$ given by

$$
Q_{n}^{*}(f ; \lambda):=\sum_{\substack{i=1 \\ i \neq k}}^{n} \bar{\mu}_{i n} \frac{f\left(\bar{x}_{i n}\right)-f(\lambda)}{\bar{x}_{i n}-\lambda}+I(\bar{w} ; \lambda),
$$


where the $\bar{\mu}_{i n}$ are the Gaussian weights and $\bar{x}_{i n}$ the Gaussian points corresponding to $\bar{w}$, that is, the zeros of $p_{n}(\bar{w} ; x)$, the polynomial orthogcnal with respect to $\bar{w}$. The index $k$ is the index of the point closest to $\lambda$. The autiors show in Theorem 2.1 and Corollary 2.3 that $Q_{n}^{*}(f ; \lambda)$ converges uniformly to $I(\bar{w} f ; \lambda)$ for all $\lambda \in(-1,1)$ if $(8)$ holds.

In [4], these same authors approximate $f$ by the Lagrange interpolating polynomial $f_{n}$ based on certain sets $X_{n}$. They show that if $x_{i n}$ are the zeros of $\left(1-x^{2}\right) p_{n-1}(\bar{w} ; x)$, then (3) holds if (8) holds. On the other hand, if $x_{i n}$ are the zeros of $p_{n+1}(\bar{w} ; x)$, then (3) holds only when $\gamma_{1}:=\min (\alpha, \beta)>0$ and $\mu+\gamma_{1}>1 / 2$.

We see that in both cases treated by these authors, the best uniform convergence results they can get require that $(8)$ hold, which is the same requirement as in Corollaries 5-7, which deal with polynomial approximations to $f$.

We conclude by remarking that Theorem 1 is also true for $I(\bar{w} f ; \lambda)$. By inspecting the proof, we see that the only thing we need worry about is the behavior of $I(\bar{w} ; \lambda)$ in the neighborhoods of \pm 1 . We show that

$$
I(\bar{w} ; \lambda)=O\left((1 \pm \lambda)^{\gamma} \log (1 \pm \lambda)\right)+C
$$

for $\lambda$ in a neighborhood of $\mp 1$, which is sufficient for our purposes.

By Lemma 5.3 in [4], in a neighborhood of $\lambda=1$,

$$
\left|I(\bar{w} ; \lambda)-\sum_{\substack{i=1 \\ i \neq k}}^{m} \frac{\bar{\mu}_{i n}}{\bar{x}_{i n}-\lambda}\right|=O \begin{cases}\left(\sqrt{1-\lambda}+m^{-1}\right)^{2 \alpha}, & \alpha>0, \\ \log \left[m^{-1}(1-\lambda)^{-1 / 2}+1\right], & \alpha=0, \\ (1-\lambda)^{\alpha}, & \alpha<0,\end{cases}
$$

for $m \geq M_{0}$, where $\bar{\mu}_{i n}, \bar{x}_{i n}$, and $k$ are as in (9). A corresponding result holds in a neighborhood of $\lambda=-1$ with $\alpha$ replaced by $\beta$. By Lemma 3.4 in [3],

$$
\left|\sum_{\substack{i=1 \\ i \neq k}}^{m} \frac{\bar{\mu}_{i n}}{\bar{x}_{i n}-\lambda}\right|=O \begin{cases}\log m, & \alpha, \beta \geq 0, \\ w(\lambda) \log m, & -1<\alpha, \beta<0,\end{cases}
$$

uniformly for $\lambda \in(-1,1)$ with similar estimates if $\alpha<0 \leq \beta$. Hence, choosing $m=M_{0}$ yields (10). Similarly, Theorem 1 is true for $I(\tilde{w} f ; \lambda)$, where $\tilde{w}(x):=\bar{w}(x)\left|\log (1-x)^{p} \log (1+x)^{q}\right|$ for any nonnegative integers $p, q$.

Note added in proof. I am indebted to Professor Philippe L. Toint for the following remarks. From Theorem 1, it appears that the rapidity of convergence of $f_{n}$ to $f$ plays a role in deciding when $I\left(w f_{n} ; \lambda\right)$ converges uniformly in $(-1,1)$ to $I(w f ; \lambda)$. However, a simple observation shows that this not the case, which implies that one can dispense with condition (b) in Theorem 1. In fact, if we write $B_{n}:=A_{n}^{\nu}$, then $B_{n}$ is also a sequence of positive numbers such that $\lim _{n \rightarrow \infty} B_{n}=0$ and $\left\|r_{n}\right\|_{\infty}=O\left(B_{n}\right)$. Since the restriction $\nu \leq \mu$ in condition (b) is never used, we have always that $\nu=1$. Hence we can replace $\rho$ by $\sigma$ and condition (b) with the hypothesis that $\left\{f_{n}\right\}$ is 
a sequence of approximations which converges uniformly to $f$ in $J$. Thus, the rate of convergence of $\left\{f_{n}\right\}$ to $f$ is irrelevant to the question of uniform convergence of $I\left(w r_{n} ; \lambda\right)$. It is only the modulus of continuity, $\omega\left(r_{n} ; t\right)$, that counts. Of course, in many cases, the Hölder index $\mu$ or $r_{n}$ depends on the rate of convergence of $r_{n}$ as in Examples 6 and 7 which use Kalandiya's Theorem to determine $\omega\left(r_{n} ; t\right)$. However, in the case of Example 5 where $\left\{f_{n}\right\}$ is the sequence of Bernstein polynomial approximations to $f$, we get a stronger result, namely, that we have uniform convergence of $I\left(w r_{n} ; \lambda\right)$ in $(-1,1)$ if $\mu+\gamma>0$ and not only for $\mu / 2+\gamma>0$.

\section{BIBLIOGRAPHY}

1. B. M. Brown, D. Elliott, and D. F. Paget, Lipschitz constants for the Bernstein polynomials of a Lipschitz continuous function, J. Approx. Theory 49 (1987), 196-199.

2. L. Brutman, On the Lebesgue function for polynomial interpolation, SIAM J. Numer. Anal. 15 (1978), 694-704.

3. C. Criscuolo and G. Mastroianni, On the uniform convergence of Gaussian quadrature rules for Cauchy principal value integrals, Numer. Math. 54 (1989), 445-461.

4. _ Mean and uniform convergence of quadrature rules for evaluating the finite Hilbert transform, submitted for publication.

5. C. Dagnino and E. Santi, On the convergence of spline product quadratures for Cauchy principal value integrals, submitted for publication.

6. P. J. Davis and P. Rabinowitz, Methods of numerical integration, 2nd ed., Academic Press, New York, 1984.

7. D. Elliott and D. F. Paget, On the convergence of a quadrature rule for evaluating certain principal value integrals: An addendum, Numer. Math. 25 (1976), 287-289.

8. G. G. Lorentz, Bernstein polynomials, Univ. of Toronto Press, Toronto, 1953.

9. Chien-Ke Lu, The approximation of Cauchy-type integrals by some kinds of interpolatory splines, J. Approx. Theory 36 (1982), 197-212.

10. E. Neuman and J. W. Schmidt, On the convergence of quadratic spline interpolants, J. Approx. Theory 45 (1985), 299-309.

11. P. Rabinowitz, Convergence results for piecewise linear quadratures for Cauchy principal value integrals, Math. Comp. 51 (1988), 741-747.

12. P. Rabinowitz and I. H. Sloan, Product integration in the presence of a singularity, SIAM J. Numer. Anal. 21 (1984), 149-166.

13. G. Szegö, Orthogonal polynomials, Amer. Math. Soc. Colloq. Publ., vol. 23, Amer. Math. Soc., Providence, RI, 1975.

14. P. Vértesi, Hermite-Fejér type interpolations. IV (Convergence criteria for Jacobi abscissas), Acta Math. Acad. Sci. Hungar. 39 (1982), 83-93.

15. P. Vértesi, personal communication.

Department of Applied Mathematics and Computer Science, The Weizmann Institute OF SCIENCE, ReHovot 76100, ISRAEL

E-mail address: maweintr.@weizmann.bitnet 